\section{Case Reports in Ophthalmology}

\title{
Sutureless 25-Gauge Pars Plana Vitrectomy Combined with Retropupillary Fixation of an Iris-Claw Intraocular Lens
}

\author{
Spyridon E. Chalkiadakis ${ }^{a, b}$ Efstratios A. Parikakis ${ }^{c} \quad$ Simon R.J. Taylor ${ }^{a, b}$ \\ ${ }^{a}$ Royal Surrey County Hospital NHS Foundation Trust, Guildford, UK; ${ }^{b}$ School of Medicine, \\ University of Surrey, Guildford, UK; ' $A$ thens Eye Hospital, Athens, Greece
}

\section{Keywords}

Pars plana vitrectomy $\cdot$ Retropupillary fixation $\cdot$ Iris-claw intraocular lens $\cdot$ Combined operation

\begin{abstract}
Background: The surgical case of a dropped intraocular lens inside the vitreous cavity constitutes a real challenge for the operating surgeon. Herein, we describe a case series where an alternative optical rehabilitation technique for late intraocular lens-bag complex dislocation has been used. Methods: A modern vitrectomy device was used to remove the capsule with the dropped intraocular lens using sutureless 25 -gauge pars plana vitrectomy. To ensure a better aesthetic result, with faster patient recovery and a reduced number of operations, the whole procedure was performed during the same operating session; an iris-claw intraocular lens for aphakia was selected for implantation. The implant was passed behind the constricted iris with the concave surface facing it. The lens was grasped with the manufacturer's holding forceps and fixed onto the posterior surface of the iris using the special enclavation needles. Results: We have operated 12 eyes in two different clinical centres successfully, with minimal intra- and/or postoperative complications. Conclusion: We believe that this is a viable solution for the visual rehabilitation of patients, who would otherwise need more than one operation for a lens exchange.

(C) 2016 The Author(s) Published by S. Karger AG, Basel
\end{abstract}




\section{Case Reports in Ophthalmology}

Chalkiadakis et al.: Sutureless 25-Gauge Pars Plana Vitrectomy Combined with Retropupillary Fixation of an Iris-Claw Intraocular Lens

\section{Introduction}

The technology related to pars plana vitrectomy (PPV) has changed dramatically since it was first introduced by R. Machemer in the early 1970s [1]. The current trend for routine cases is to operate using the smallest instrument gauge possible. The reasons for that are numerous and related to less surgical trauma and more sophisticated intraocular fluidics. Moreover, the stiffness of the current instruments allows for the same manoeuvrability with small-gauge instruments as with larger ones without any detriment to patient safety. Therefore, the recovery of dislocated intraocular lenses (IOLs) can be safely accomplished with the accompanying small-gauge surgical forceps. In cases of severe pseudoexfoliation the chances of late IOL dislocation inside the vitreous cavity constitute a significant postoperative complication. After a successful small-gauge PPV and removal of the IOL, the options for visual recovery vary. An anterior chamber IOL can be chosen in selected cases where other factors of co-morbidity do not exist, such as glaucoma or a low endothelial cell count. Another option is a scleral sutured IOL. Nevertheless, this type of implantation demands complex surgical manipulations with possible intraoperative complications.

An alternative choice is the iris-claw IOL. In our cases we used the Ophtec Artisan lens for aphakia, a single-piece, non-foldable acrylic lens. In this method, the lens is not supported anymore by the angle of the anterior chamber but by the stroma of the iris, after a special insertion manoeuvre called enclavation.

Moreover, reports have emerged of late corneal decompensation after the implantation of these lenses in the anterior chamber [2,3]. For this reason, we believe that the technique of retropupillary insertion of iris-claw lenses, first described by Amar [4], could become even more relevant. The study and data accumulation were carried out after prospective approval from the Departmental Ethics Committees of the Athens Eye Hospital, Athens, Greece, and the Royal Surrey County Hospital NHS Foundation Trust, Guildford, UK, respectively. It was funded by the National Health Systems of both countries. The end point of the study, entitled 'Proof of concept of the retropupillary insertion of the iris-claw lens after pars plana vitrectomy', was to prove the safety of the technique and the financial benefit for the Trusts, since the entire process is concluded in one operating session.

\section{Technique}

The procedure is performed under local (parabulbar) anaesthesia with a mixture of $50 \%$ ropivacaine hydrochloride solution and $50 \%$ lidocaine solution $2 \%$; the eye is dilated, when possible, through the topical instillation of drops of tropicamide 1 and $2.5 \%$ phenylephrine hydrochloride solution. Once the 25-gauge PPV is concluded, trocars are left in place to take advantage of the excellent fluidics control of the new generation of vitrectomy machines. In this manner the vitreous cavity remains pressurised and there is an immediate system response to abrupt intraocular pressure variations. Therefore, the danger of intraoperative complications such as hypotony, extrachoroidal haemorrhage, or iris prolapse is limited.

After the dislocated lens (fig. 1) and the remaining capsule have been brought into the anterior chamber of the eye (fig. 2), they are extracted through a 4-mm corneal incision, under the protection of a layer of a dispersive ophthalmic viscosurgical device (OVD), which is injected to protect the corneal endothelium. 
The anterior chamber is re-filled with the dispersive OVD, and two side ports are performed at 3 and 9 o'clock. The new lens is inserted through the corneal incision with the convex part facing the posterior pole to avoid pupillary block. Safety sutures are used on the corneal incision, and the lens is rotated to achieve a horizontal orientation. The original industrial holding forceps of the lens are inserted inside the anterior chamber, and the IOL is grasped. With a careful diving technique, the lens haptics are sequentially brought behind the iris plane (fig. 3). The pupil is brought down with an intracameral injection of acetylcholine chloride solution. The first haptic of the IOL is fixed onto the posterior stroma of the iris using the special enclavation needle (fig. 4a); then, the surgeon alternates the hands holding the forceps, and the second haptic is also enclavated (fig. 4b). The anterior chamber is washed using the bimanual technique (fig. 5). The side ports are hydrated, and the corneal incision is checked for watertightness (fig. 6) (please watch online suppl. video; for all online suppl. material, see www.karger.com/doi/10.1159/000448943).

\section{Discussion}

New-generation vitrectomy machines offer excellent fluidics control during each step of a procedure. Using special sensors, the machine can control the intraocular pressure instantly and thereby equilibrate intraoperative variations without causing any damage to the ocular structures. Because of this, the operating surgeon can perform demanding intraocular manoeuvres in a steady environment, even in the presence of open corneal or scleral incisions.

Our initial experience was with a 20-gauge operating machine that supported a vented gas-forced infusion system; in these procedures, we felt that the iris plane was unstable during IOL enclavation and that the learning curve could be very steep. With small-gauge PPV, the vitrectomy machine was found to provide a more stable anterior chamber, even with open sclerotomies, because it has an intraocular pressure compensation system that compensates for pressure drops in the infusion tubing and cannula.

Retropupillary iris-claw lens implantation has the advantage of preserving the normal anatomy of the eye and being close to the eye's nodal point. Mohr et al. [5] were the first to study a large consecutive cohort of patients with this method of implantation during secondary insertion of the lens for the correction of aphakia. The authors calculated an estimated optical A-constant of 116.8, which was included in the software of modern optical machines for biometry and confirmed by the work of Wolter-Roessler and Küchle [6]. Moreover, Gicquel et al. [7] studied higher-order aberrations from the retropupillary insertion of irisclaw lenses. They concluded that such aberrations were less than those originating from the fixation of the lens in the anterior chamber of the eye. Regarding the insertion technique itself, a good synchronisation is needed between the primary surgeon and the assistant at the critical point of hand alternation.

The probability of late lens subluxation was not considered high enough to be relevant, because it is possible to confirm the enclavation during the insertion manoeuvre and predict its efficiency. During this procedure, the official manufacturer's tools accompanying the lens were used, and we do not have an opinion regarding the newer tools engineered by Prof. Sekundo [8].

The proposed method is in agreement with previous studies stating that the lens should be inserted in the plano-convex direction. In such a way, the possibilities of cases with pigment dispersion, hyphaema, or late uveitis are limited. The pupil can become oval some- 
times, but the incidence of postoperative cystoid macular oedema was not different from that after routine cases of in-the-bag IOL implantation. No cases of postoperative pressure spikes were noticed, and a peripheral iridectomy was not considered necessary, the reason being the aforementioned lens configuration that leaves enough space between the lens and the pupil without a risk of pupillary block. In our case series of 12 operated patients, we only had 1 case that needed reenclavation of one of the IOL haptics a few months after the original operation.

In conclusion, the management of cases with serious pseudoexfoliation and IOL dislocation appears to be challenging. Small-gauge PPV, combined with controlled implantation of an iris-claw IOL behind the iris plane during the same operating session, is a safe alternative.

\section{Statement of Ethics}

The study followed the tenets of the Declaration of Helsinki, and informed consent for the research was obtained from all participants at the beginning of each operation.

\section{Disclosure Statement}

The authors have no financial or proprietary interest in a product, method, or material described herein. The authors did not receive any financial support from any public or private sources.

\section{References}

1 Machemer R, Buettner H, Norton EW, Parel JM: Vitrectomy: a pars plana approach. Trans Am Acad Ophthalmol Otolaryngol 1971;75:813-820.

-2 van Eijden R, de Vries NE, Cruysberg LP, Webers CA, Berenschot T, Nuijts RM: Case of late-onset corneal decompensation after iris-fixated phakic intraocular lens implantation. J Cataract Refract Surg 2009;35:774-777.

-3 Kim M, Kim JK, Lee HK: Corneal endothelial decompensation after iris-claw phakic intraocular lens implantation. J Cataract Refract Surg 2008;34:517-519.

$4 \quad$ Amar L: Posterior chamber iris claw lens. Am Intraocul Implant Soc J 1980;6:27.

-5 Mohr A, Hengerer F, Eckardt C: Retropupillary fixation of the iris claw lens in aphakia. 1 year outcome of a new implantation technique (in German). Ophthalmologe 2002;99:580-583.

6 Wolter-Roessler M, Küchle M: Correction of aphakia with retroiridally fixated IOL (in German). Klin Monbl Augenheilkd 2008;225:1041-1044.

7 Gicquel JJ, Nochez Y, Pisella PJ, Dighiero PL: Evaluation of the higher order wavefront aberrations and the quality of vision after correction of aphakia by a retropupillary implantation of the Verisyze ${ }^{\circledR}$ irisclaw intraocular lens. European Association for Vision and Eye Research - EVER 2010 Congress.

-8 Sekundo W: New forceps and spatula for easy retropupillary implantation of iris claw lenses in aphakia: experience in 4 years of use. Eur J Ophthalmol 2008;18:442-444. 


\section{Case Reports in Ophthalmology}

\begin{tabular}{l|l}
\hline Case Rep Ophthalmol 2016;7:155-161 \\
\hline DOI: 10.1159/000448943 & $\begin{array}{l}\text { C } 2016 \text { The Author(s). Published by S. Karger AG, Basel } \\
\text { www.karger.com/cop }\end{array}$ \\
\hline
\end{tabular}

Chalkiadakis et al.: Sutureless 25-Gauge Pars Plana Vitrectomy Combined with Retropupillary Fixation of an Iris-Claw Intraocular Lens

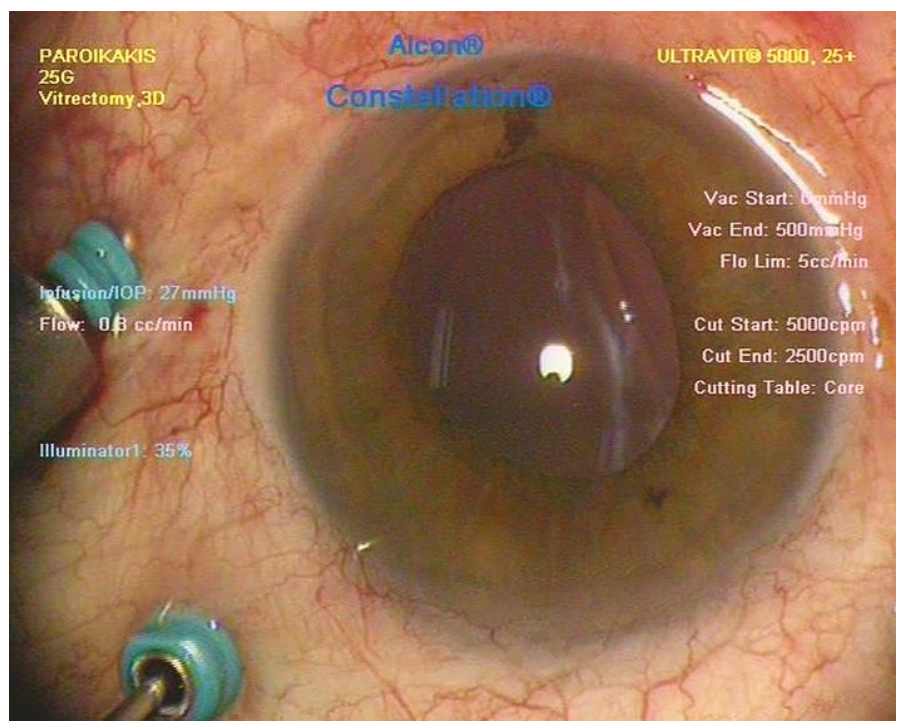

Fig. 1. Our case during small-gauge vitrectomy, where the late IOL-bag dislocation is evident.


Fig. 2. The IOL is grasped with a 25 -gauge inner limiting membrane forceps (a) and brought into the anterior chamber (b). 


\section{Case Reports in Ophthalmology}

\begin{tabular}{l|l}
\hline Case Rep Ophthalmol 2016:7:155-161 \\
\hline DOI: 10.1159/000448943 & $\begin{array}{l}\text { C } 2016 \text { The Author(s). Published by S. Karger AG, Basel } \\
\text { www.karger.com/cop }\end{array}$ \\
\hline
\end{tabular}

Chalkiadakis et al.: Sutureless 25-Gauge Pars Plana Vitrectomy Combined with Retropupillary Fixation of an Iris-Claw Intraocular Lens

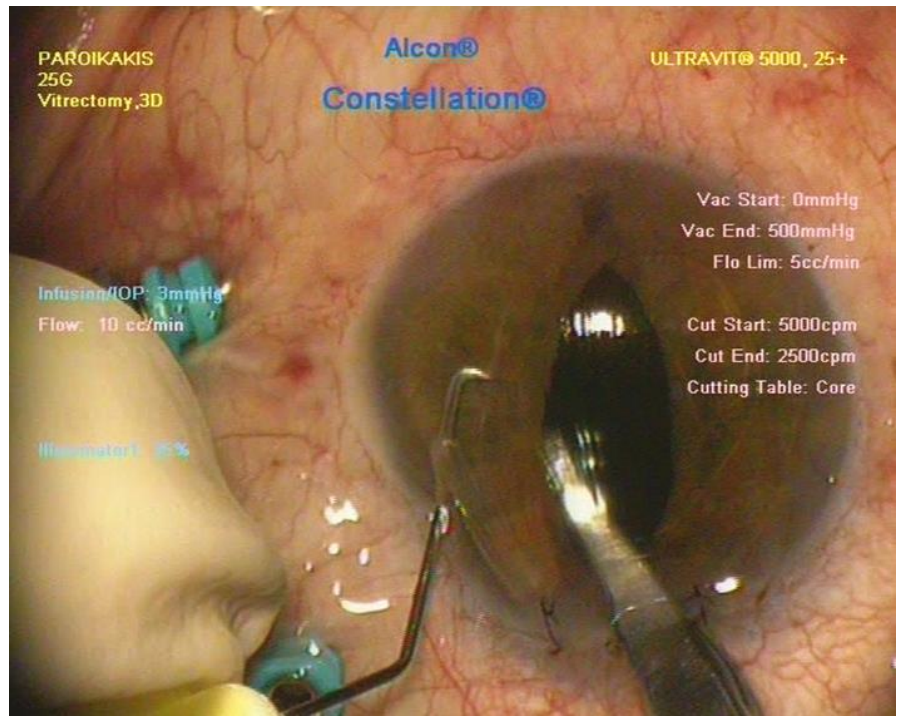

Fig. 3. The iris-claw lens is inserted behind the iris using a special holding forceps.
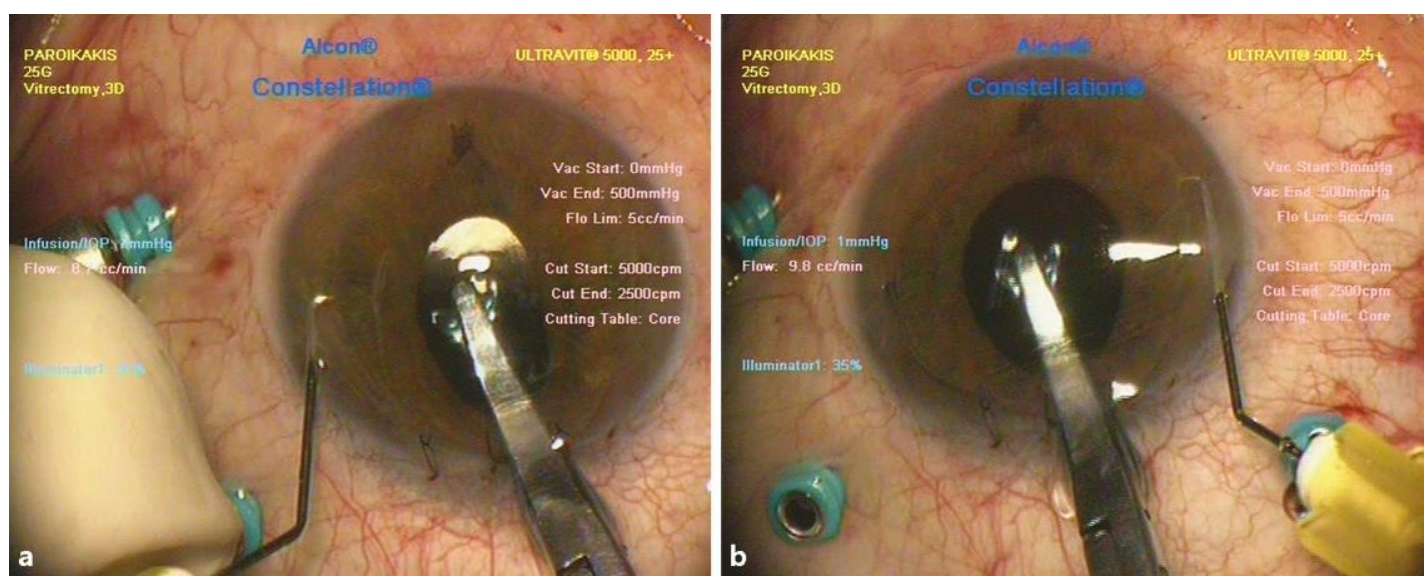

Fig. 4. a Holding the iris-claw lens behind the iris, the left haptic is enclavated onto the posterior surface of the iris. $\mathbf{b}$ Right haptic fixation onto the posterior surface of the iris. The enclavation needle can be seen to the right. 


\section{Case Reports in Ophthalmology}

\begin{tabular}{l|l}
\hline Case Rep Ophthalmol 2016;7:155-161 \\
\hline DOI: 10.1159/000448943 & $\begin{array}{l}\text { @ 2016 The Author(s). Published by S. Karger AG, Basel } \\
\text { www.karger.com/cop }\end{array}$ \\
\hline
\end{tabular}

Chalkiadakis et al.: Sutureless 25-Gauge Pars Plana Vitrectomy Combined with Retropupillary Fixation of an Iris-Claw Intraocular Lens

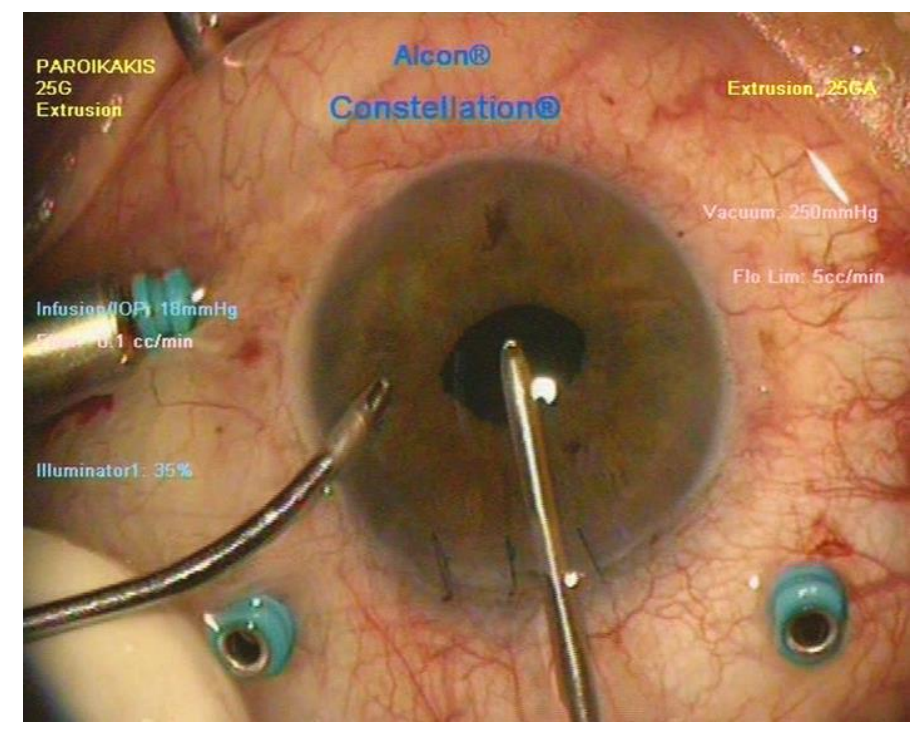

Fig. 5. The OVD is washed through using the bimanual technique.

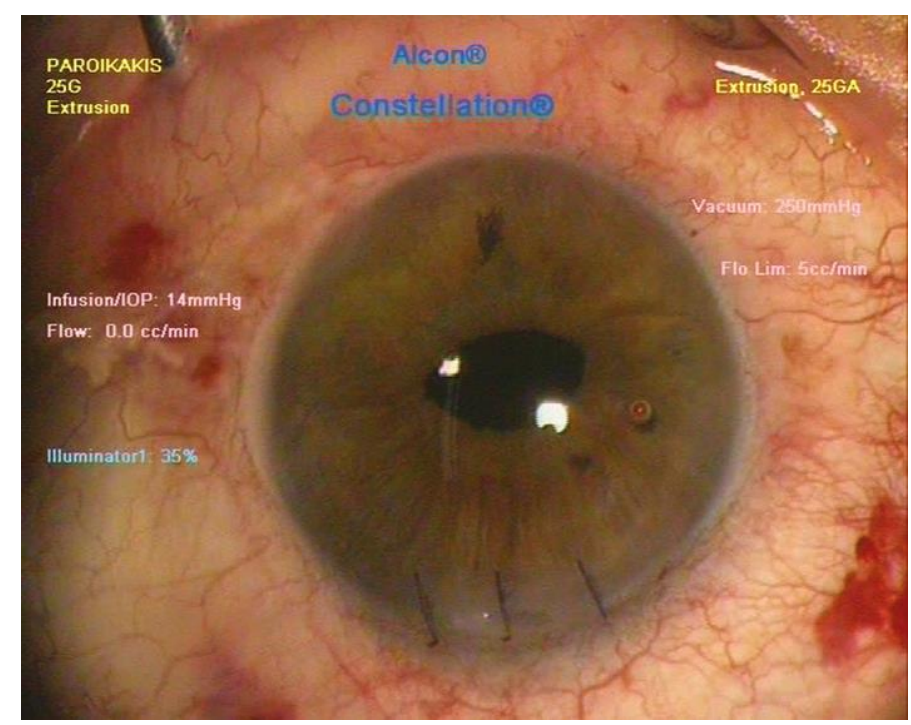

Fig. 6. The operation is completed with a round pupil and the iris-claw lens well centred behind the iris. 